Int. J. Dev. Biol. 49: 895-899 (2005)

doi: $10.1387 / \mathrm{ijdb} .051980 \mathrm{rt}$

Developmental Expression Pattern

\title{
Posterior expression of nanos orthologs during embryonic and larval development of the anthozoan Nematostella vectensis
}

\author{
RAQUEL TORRAS and SERGIO GONZÁLEZ-CRESPO* \\ Institut de Biologia Molecular de Barcelona, CSIC, Barcelona, Spain
}

\begin{abstract}
Cnidarians are primitive animals located in a basal position in the phylogenetic tree of the Animal Kingdom, as an outgroup of the Bilaterians. Therefore, studies on cnidarian developmental biology may illustrate how fundamental developmental processes have originated and changed during animal evolution. A particular example of this is the establishment of polarity along the body axes, which is under the control of a number of developmental genes, most of them conserved in evolution and playing similar roles in diverged species. Concerning the anteriorposterior axis, genetic and molecular studies on Drosophila have shown that the nanos gene plays an essential role in defining posterior structures during early embryonic development. Here we report the isolation of two nanos orthologs in the anthozoan Nematostella vectensis. We show that nanos mRNA is asymmetrically distributed in the fertilized egg and this asymmetry is maintained during embryonic development. At gastrula and planula larva stages, nanos expression is permanently associated with posterior body regions. These results, together with our previous analysis in the hydrozoan Podocoryne carnea, indicate that posterior nanos expression during development is a conserved feature among cnidarians. Therefore, the potential role of cnidarian nanos in defining axial polarity as a posterior determinant would represent an ancestral trait in the Animal Kingdom.
\end{abstract}

KEY WORDS: Cnidaria, planula, anterior-posterior axis, axial patterning, evolution

A major topic in developmental biology is the study of the origin and evolution of the genetic mechanisms responsible for the specification of animal body axes. This process has been deeply investigated in Drosophila, where a number of genes responsible for anterior-posterior (A-P) patterning have been identified (reviewed in Gilbert, 2003). Essentially, maternal gene products that appear differentially localized in the early embryo regulate the expression of zygotic segmentation genes, which, ultimately, define the domains of Hox gene expression. Hox products confer positional identity to the different embryonic and adult territories along the A-P axis (reviewed in Gilbert, 2003). An example of an early-acting gene that participates in the specification of A-P polarity is nanos, whose maternal product is a CCHC-type $\mathrm{Zn}$ finger protein localized to the posterior region of the early Drosophila embryo (Wang and Lehmann, 1991). nanos is essential for the appropriate formation of posterior structures, namely the abdomen (Wang and Lehmann, 1991). Additionally, nanos plays a role during the differentiation of the Drosophila germline (Kobayashi et al., 1996).
Most of these Drosophila axial patterning genes are conserved and play related functions in different animal groups, so they can be studied to trace the evolution of the process throughout the phylogenetic tree of the Animal Kingdom. In this tree, the phylum Cnidaria appears located at a basal position, as outgroup of the Bilateria (Adoutte et al., 2000), so its study might provide significant insights on how basic animal body plans have originated and evolved at the base of the tree.

In this regard, we recently found that in the hydrozoan Podocoryne carnea, nanos orthologs are transiently expressed at the posterior pole of the early embryo (Torras et al., 2004), raising the possibility that they are involved in the control of axial patterning, in a similar way as in Drosophila. To determine if this is a conserved feature among cnidarians, we decided to analyze nanos expression during development of the anthozoan Nematostella vectensis.

The starlet sea anemone Nematostella vectensis (Hand and

Abbreviations used in this paper: AP, anterior-posterior.

\footnotetext{
*Address correspondence to: Dr. Sergio González Crespo. Institut de Biologia Molecular de Barcelona, CSIC. Barcelona Science Park, Josep Samitier, 1-5. 08028 - Barcelona, Spain.Fax: +34-93-403-4979. e-mail: sgcbmc@cid.csic.es
} 
Uhlinger, 1992) is progressively emerging as a useful model system to approach questions related to evolutionary developmental biology. Among the cnidarians, anthozoans are considered primitive, since they only have one of both cnidarian adult body forms, namely the polyp, while the medusa is missing (reviewed in Brusca and Brusca, 2003). Also, molecular phylogenetic analyses suggest that anthozoans are basal cnidarians (Bridge et al., 1995). In Nematostella, both sexes are separated and adult polyps release either male or female gametes to the water. The fertilized egg undergoes a variable pattern of cleavage that results into a hollow blastula. Next, during the process of gastrulation, cells located at the posterior pole of the blastula invaginate and the blastopore forms. These invaginating cells finally fill up the internal cavity and give rise to the endoderm, whereas the external cells become ciliated and form the ectoderm (Hand and Uhlinger, 1992). This bi-layered developmental stage corresponds to the canonical cnidarian planula larva (Brusca and Brusca, 2003). The Nematostella planula swims actively, always with the same pole to the front. This pole, referred to as anterior, is easily recognizable by an apical sensory tuft of large cilia. The posterior pole of the planula corresponds to the region where the blastopore was located at the gastrula stage (Hand and Uhlinger, 1992). A few days later, the planula settles and transforms into a juvenile polyp by formation of four major structures, i. e.: 1) a gastrovascular cavity, called coelenteron, that opens in the endo- derm, 2) a mouth with a pharynx formed by invagination of the posterior ectodermal region, 3) a number of endoderm projections, called mesenteries, that connect with the pharynx and 4) four tentacles surrounding the oral opening (Hand and Uhlinger, 1992; Martindale et al., 2004; Finnerty et al., 2004). Therefore, with respect to the polarity of the body axis, the posterior region of the planula gives rise to the mouth of the polyp. Since this significant feature has been also observed in hydrozoans (Freeman, 1981; Schwoerer-Böhning et al., 1990), the correspondence between the larval A-P axis and the aboral-oral axis in the adult polyp is considered a general feature of cnidarian development. Remarkably, the juvenile Nematostella polyp moves in the opposite direction than the planula (Hand and Uhlinger, 1992; our observations), that is, with its mouth to the front. In other words, during the transition from planula to polyp there is an inversion of the swimming direction.

Here we provide evidence that nanos expression in Nematostella is restricted to posterior precursors throughout embryonic and larval development. First, we show that maternal nanos mRNA is localized to one side of the fertilized egg. During early embryogenesis, nanos expression remains restricted to approximately half of the blastomers located at one side of the cleaving embryo. In the gastrula, only cells located in the anterior pole and those that invaginate do not show nanos expression. In the planula larva, nanos mRNA accumulates preferentially in the

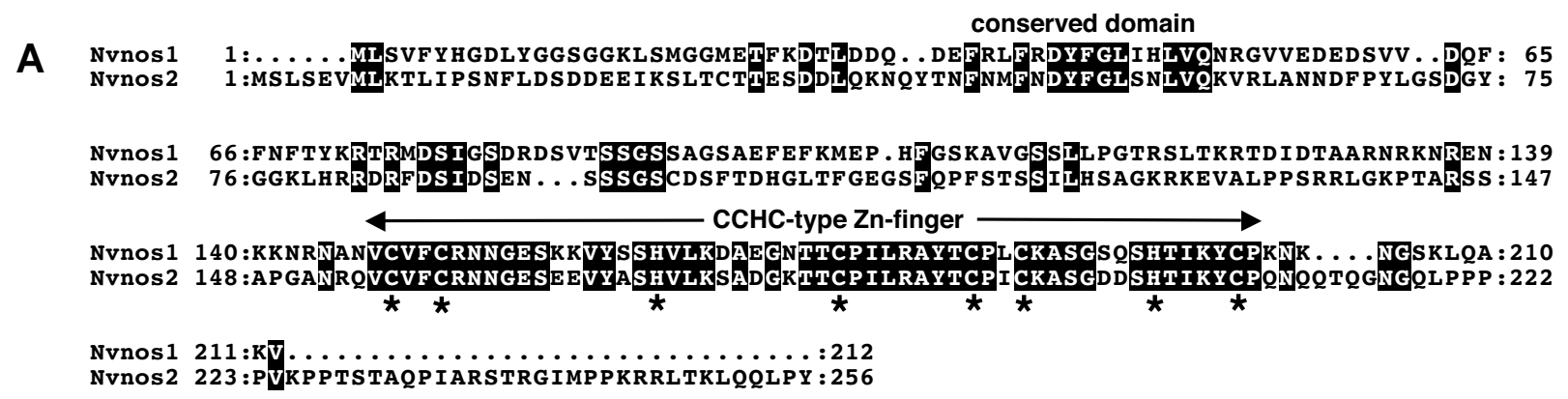

B

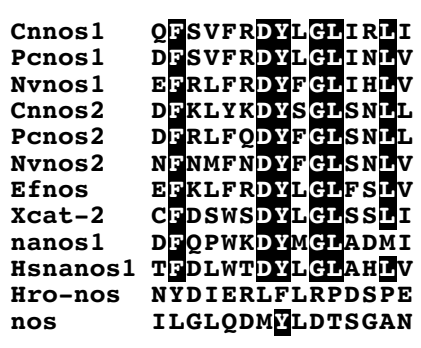

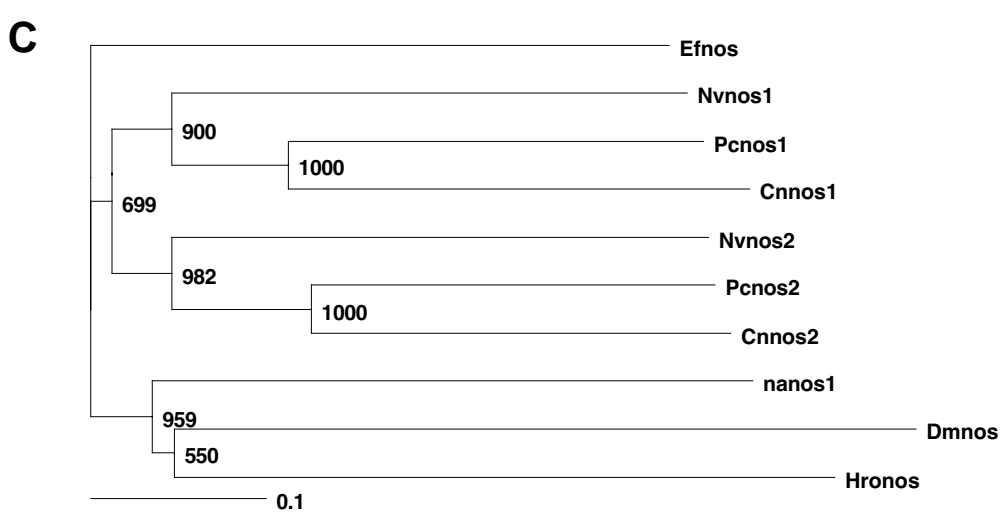

Fig. 1. Nvnos1 and Nvnos2 sequence analysis. (A) Deduced amino acid sequence of Nematostella nanos cDNAs (Nvnos1 and Nvnos2) aligned to each other. The CCHC-type Zn-finger domain and the upstream conserved domain are indicated. Conserved Cs and Hs are indicated by stars. (B) Presence of the upstream conserved domain in non-protostome animals. Sequences are from the cnidarians Hydra (Cnnos1 and Cnnos2), Podocoryne (Pcnos1 and Pcnos2) and Nematostella (Nvnos1 and Nvnos2), the sponge Ephydatia fluviatilis (Efnos), the deuterostomes Xenopus (Xcat-2), zebrafish (nanos1), human (Hsnanos1) and the protostomes Helobdella robusta (Hro-nos) and Drosophila (nos) (refs. in Torras et al., 2004). (C) Phylogenetic analysis of full-length Nanos proteins. Gene abbreviations as in B. Numbers in the branches correspond to bootstrap replicates out of 1000 supporting the topology shown. Efnos sequences were defined as outgroup and used to root the tree. 

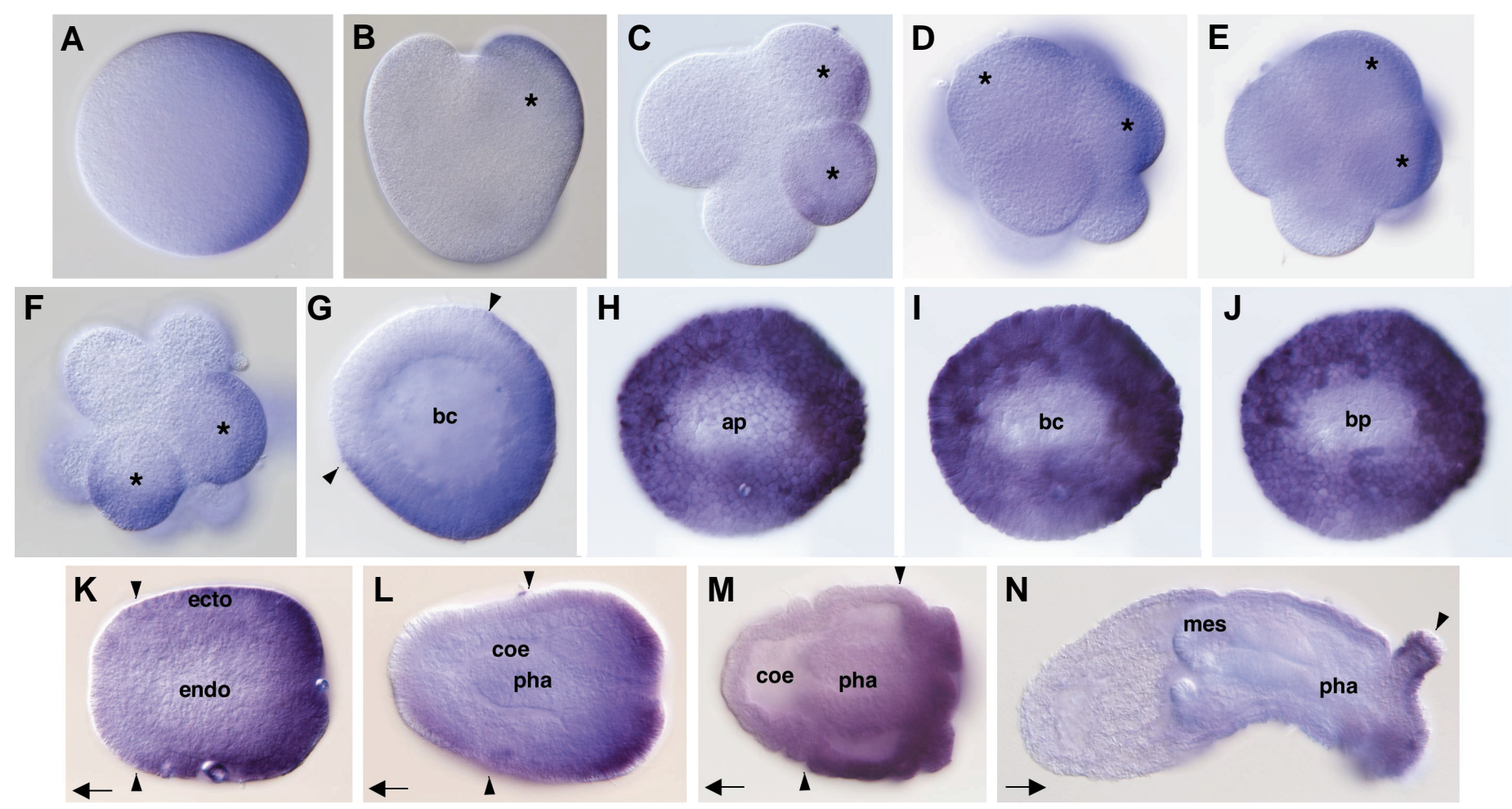

Fig. 2. Nvnos1 expression pattern during Nematostella development. (A) Fertilized oocyte stained for Nvnos1 mRNA. Staining forms a gradient with peak levels in one side that decrease towards the centre. This pattern is consistently observed in nearly all embryos examined. No staining is detected using sense riboprobes. (B-F) Cleaving embryos. Cells containing Nvnos1 mRNA are indicated by stars. (B) Beginning of the 2-cell stage. Nvnos1 expression is detected in one of the cells but not in the other. (C) 4-cell stage. Only two adjacent cells contain Nvnos1 mRNA. (D,E) Same 8-cell stage embryo shown at two different focal planes. In both cases, Nvnos1 expression is localized in two adjacent cells. (F) 12-cell embryo showing Nvnos1 expression. (G) Hollow blastula. Nvnos1 expression is localized to one side and extends to the limits indicated by arrowheads. (H-J) Same early gastrula stained for Nvnos1 shown at three optical focal planes. (H) View at the level of the anterior pole (ap). Note that anterior unstained cells appear at the same focal plane that surrounding stained cells. (I) Optical middle section. The empty hole corresponds to the blastocoele (bc). (J) View at the blastopore (bp) level. Note that unstained invaginating cells are slightly out of focus in relation to the surrounding stained cells. (K-M) Lateral views of planulae oriented with anterior to the left and posterior to the right. The anterior apical sensory tuft of large cilia is lost during the process of in situ hybridization. Arrowheads indicate the approximate anterior limits of gene expression. (K) Nvnos1 expression. Staining accumulates preferentially in the ectoderm of the posterior region. (L) Older planula showing Nvnos1 staining. Note the forming coelenteron and pharynx. Although Nvnos1 expression is still posteriorly localized, some asymmetry is observed. (M) Old planula showing sustained Nvnos1 expression in the posterior region. (N) Juvenile polyp. Nvnos1 expression is limited to the tentacle buds (arrowhead). Abbreviations: ap, anterior pole; bc, blastocoele; bp, blastopore; coe, coelenteron; ecto, ectoderm; endo, endoderm; mes, mesenteries; pha, pharynx. Swimming direction is indicated by an arrow at the bottom left of the figures.

posterior region. Finally, posterior nanos expression disappears when the swimming polarity reverses and the primary polyp forms. Altogether, these results indicate a conservation of posterior nanos expression during early cnidarian development and suggest an ancient involvement of nanos in axial patterning as a posterior determinant.

\section{Results and Discussion}

To study the ancestry of nanos expression in posterior embryonic regions, we have isolated corresponding orthologs in the anthozoan Nematostella vectensis and analyzed their expression patterns throughout development. We have isolated two Nematostella nanos orthologs, Nvnos1 and Nvnos2, encoding proteins of 212 and 256 amino acid residues, respectively (Fig. $1 \mathrm{~A})$. Both proteins contain a domain with two CCHC-type Znfingers highly similar to those present in Nanos proteins from other species (not shown). Also, they show a short region close to the amino-terminus that is conserved in other animals with the exception of the protostomes (consensus FxxxxDYxGLxxL) (Fig. $1 \mathrm{~A}, \mathrm{~B})$. The functional significance of this region is presently unknown. We also performed phylogenetic analysis with the complete Nanos amino acid sequences from Nematostella, Hydra, Podocoryne, the sponge Ephydatia, the leech Helobdella, Drosophila and zebrafish (refs. in Torras et al., 2004) using the neighbor-joining method. In the resulting tree (Fig. 1C), the cnidarian type 1 nanos genes group with themselves with preference to type 2 genes from the same species, thus suggesting that both cnidarian nanos genes arose from an ancestral duplication that predated cnidarian diversification, as previously suggested by analysis of the $\mathrm{Zn}$-finger domain (Torras et al., 2004).

Next, we analyzed the expression of Nvnos1 and Nvnos2 during Nematostella development by RT-PCR (data not shown). We found that Nvnos1 mRNA is already present in very early embryos and is probably of maternal origin. The levels of Nvnos 1 
mRNA remain fairly constant throughout embryonic and larval development. On the contrary, Nvnos2 expression is firstly detected at gastrula stages, so there is no maternal contribution for this transcript. Both Nvnos1 and Nvnos2 showed similar levels of expression at gastrula and planula stages.

We also analyzed Nvnos 1 and Nvnos2 expression patterns during Nematostella embryonic and larval development by wholemount in situ hybridization. Contrary to ubiquitous nanos expression in Podocoryne early embryos (Torras et al., 2004), Nvnos 1 mRNA of maternal origin appears localized to one side of the fertilized egg (Fig. 2A). This asymmetrical distribution is maintained during 2 and 4-cell stages, where Nvnos1 mRNA is detected only in one half of the embryo (Fig. 2B, C). Although cleavage in Nematostella embryos does not follow a stereotyped pattern (Martindale etal., 2004), we always detect Nvnos 1 mRNA in contiguous cells located at one side of the cleaving embryo (Fig. 2D-F). In the resulting hollow blastula, Nvnos1 expression is present throughout approximately one half of the embryo (Fig. $2 \mathrm{G}$ ). Due to the spherical shape of the blastula, it is difficult to determine precisely its orientation. Nevertheless, this localized Nvnos 1 expression is sustained in immediately following stages, which can unambiguously be oriented; since in these embryos Nvnos1 is posteriorly localized (see below), we assume that its expression in the blastula corresponds to the presumptive posterior region.

Therefore, the restriction of nanos expression to posterior regions of the cleaved embryo is essentially conserved in both cnidarian species Podocoryne (hydrozoan) and Nematostella (anthozoan). The distribution of maternal Nvnos1 mRNA in the Nematostella zygote (Fig. 2A) is closely reminiscent of nanos expression in the Drosophila freshly laid egg (Wang and Lehmann, 1991). In Drosophila, untranslated sequences present in maternal nanos mRNA are responsible for its anchoring to the embryonic posterior pole (Gavis et al., 1996). It is possible that a conserved mechanism could mediate Nvnos 1 mRNA localization in Nematostella embryos, although we have not detected any particular sequence similarity in the untranslated regions. In Podocoryne, the restriction of nanos mRNA to posterior regions is a later event, firstly detected in the blastula (Torras et al., 2004).

At the early gastrula stage, the embryonic A-P axis can be morphologically recognized for the first time, since the blastopore, which forms at the posterior pole, is easily visible. By this stage, Nvnos 1 is highly expressed throughout most of the embryo (Fig. 2I), but not in the anterior pole (Fig. $2 \mathrm{H}$ ). Also, invaginating cells at the blastopore region do not show Nvnos 1 expression (Fig. 2J). This result indicates that the expression observed at the blastula (Fig. 2G) (and probably at previous stages) corresponds to presumptive posterior regions. Again, this pattern is well conserved when compared to nanos expression in the Podocoryne gastrula, which remains restricted to the posterior region (Torras et al., 2004). Moreover, in both species, invaginating cells do not show nanos expression (Fig. 2J; Torras et al., 2004). However, in Nematostella the posterior domain of expression is largely expanded when compared to Podocoryne, thus leaving a relatively small anterior region without nanos expression (Fig. $2 \mathrm{H}$ ).

During development of the planula larva, Nvnos 1 expression remains preferentially localized to posterior regions of the ectoderm (Fig. 2K). A similar expression pattern is observed for Nvnos2 at this stage (data not shown). Contrary to Nvnos1,
Nvnos2 expression is firstly detected at early gastrula stages both by RT-PCR and in situ hybridization (data not shown), so its origin is exclusively zygotic. A significant difference between Nematostella and Podocoryne is the persistence of Nvnos 1 and Nvnos2 expression in planula stages, because in Podocoryne both orthologs are totally silent (Torras et al., 2004).

At the time when the coelenteron, the pharynx and the mesenteries form, Nvnos1 expression is maintained at the posterior ectoderm with a certain asymmetry (Fig. 2L, M). Finally, when the juvenile polyp forms and the swimming direction is reversed, nanos expression disappears from the epidermal body trunk; we only observe Nvnos1 staining in the developing tentacles (Fig. 2N). Similar results were obtained for Nvnos2 (data not shown). The fact that both Nvnos 1 and Nvnos2 show similar expression patterns throughout development raises the possibility of functional redundancy. Alternatively, since both genes have been maintained after such an early duplication, it is conceivable that they may play independent functions.

In the juveniles, no nanos expression is detected in the mesenteries, where the gonads will develop in the adult animal. Since nanos expression in developing germ cells is well conserved throughout the Animal Kingdom, including the cnidarians (Mochizuki et al., 2000; Torras et al., 2004), we suggest that Nematostella germline differentiation takes place in later stages of development, as it is the case for Podocoryne (Torras et al., 2004). In this regard, we detected Nvnos 1 and Nvnos2 expression in both aboral and oral regions of the adult polyp by RT-PCR (data not shown), probably associated to the developing germline cells. The fact that, in addition to nanos, cnidarian developing germ cells show conserved expression of other germline-specific genes such as vasa (Mochizuki et al., 2001) and Piwi (Seipel et al., 2004), as in Bilateria, suggests that germline development in Metazoa is a homologous process.

Together with our previous studies in Podocoryne (Torras et al., 2004), these results indicate that the expression of nanos in developing posterior precursors is a conserved feature in the phylum Cnidaria. Due to the basal phylogenetic position of this phylum, a possible function of nanos as a posterior determinant in Nematostella and Podocoryne would represent an ancestral trait in the Animal Kingdom. Cnidarian nanos genes could regulate the formation of posterior/oral structures, such as the mouth or the tentacles.

If nanos plays a role as a posterior determinant in cnidarians, its expression domain should be in agreement with the distribution of the different cnidarian Hox genes along the A-P axis. Unfortunately, there is no obvious conservation between the reported Hox expression domains for Nematostella (Finnerty et al., 2004) and Podocoryne (Yanze et al., 2001). For example, in Nematostella, the expression of anthox1 (an ortholog of the Drosophila "posterior" Hox gene $A b d B$ (Finnerty et al., 2004)) is localized to the anterior pole during blastula and gastrula stages, in a domain complementary to nanos expression. However, in Podocoryne the anterior pole is the domain of expression of Cnox1-PC, an ortholog of the Drosophila "anterior" Hox gene labial (Yanze et al., 2001).

In spite of the lack of conservation of Hox expression between Nematostella and Podocoryne, posterior nanos expression is well conserved in both cnidarian species. In this scenario it is conceivable that Hox genes may be dispensable for the formation 
of the A-P axis during early cnidarian development, as it has been suggested in C. elegans (Wang et al., 1993), the leech (NardelliHaefliger et al., 1994) and the sea urchin (Arenas-Mena et al., 1998). At early stages, Nematostella and Podocoryne nanos could be responsible for posterior patterning. Later, when the planula develops into a juvenile polyp and nanos expression disappears from the posterior epidermis, Hox genes could exert their role in regulating axial polarity. Then, for example, the aboral tip could be patterned under anthox 1 regulation, thus acquiring a "posterior" identity coincident with the time of the switch in the swimming direction.

\section{Experimental Procedures}

\section{Animal culture}

Nematostella vectensis animals were provided by Dr. Ulrich Technau (Sars Centre for Marine Molecular Biology, Norway). They were kept in filtered $33 \%$ artificial seawater at $17^{\circ} \mathrm{C}$ and fed with freshly hatched Artemia nauplius larvae three times per week. The medium was changed once a week. Spawning was induced by a feeding/starvation regime, heat and light, as described (Fritzenwanker and Technau, 2002). Fertilized eggs, embryos and planula larvae were collected for in situ hybridization.

\section{Polymerase chain reaction and cDNA cloning}

RT-PCR fragments encoding the nos $\mathrm{Zn}$-finger domain were obtained from RNA from Nematostella polyps using the oligonucleotides 5'CGGAATTCCGTGYGTNTTYTGYVRNAAYAA-3' and 5'CGGGATCCCGGGRCARTAYTTNAYNGTRTG-3' in 30 cycles at $43^{\circ} \mathrm{C}$ as annealing temperature. Corresponding full-length cDNAs were isolated from a cDNA library provided by Dr. John Finnerty (Boston University) by $\mathrm{PCR}$ using specific oligonucleotides from the $\mathrm{Zn}$-finger region and vector sequences. Both DNA strands were sequenced by using the $A B I$ 377 analyzer (Perkin Elmer) and Big Dye (Applied Biosystems) terminator chemistry.

\section{Phylogenetic analysis}

Sequence alignments and neighbour-joining analysis were done using the Clustal $X$ and Tree-View programs. Similar results were obtained using maximum parsimony and maximum likelihood methods with the PAUP and Tree-Puzzle programs, respectively (data not shown).

\section{Whole-mount in situ hybridization}

Fertilized eggs, embryos and planula larvae were processed for whole-mount in situ hybridization as described (Scholz and Technau, 2003).

\section{Acknowledgements}

We thank Jaume Baguñà, Emili Saló and Gerardo Jiménez for comments on the manuscript, Ulrich Technau for Nematostella animals and a detailed in situ protocol and John Finnerty for a Nematostella CDNA library. $R . T$. is a predoctoral fellow from the Spanish Ministerio de Educación y Ciencia. This work was supported by a grant from the Spanish Ministerio de Educación y Ciencia (BMC2001-0566) to S. G. -C.

\section{Note added in Proof:}

Nvnos 1 and Nvnos2 sequences have been deposited in GenBank under the accession numbers DQ066724 and DQ066725, respectively.

\section{References}

ADOUTTE, A., BALAVOINE, G., LARTILLOT, N., LESPINET, O., PRUD'HOMME, B. and DE ROSA, R. (2000). The new animal phylogeny: Reliability and implications. Proc Natl Acad Sci USA 97: 4453-4456.
ARENAS-MENA, C., MARTINEZ, P., CAMERON, R.A. and DAVIDSON, E.H. (1998). Expression of the Hox gene complex in the indirect development of a sea urchin. Proc Natl Acad Sci USA 95: 13062-13067.

BRIDGE, D., CUNNINGHAM, C.W., DESALLE, R. and BUSS, L.W. (1995). Classlevel relationships in the phylum Cnidaria: Molecular and morphological evidence. Mol Biol Evol 12: 679-689.

BRUSCA, R.C. and G.J. BRUSCA. (2003). Invertebrates. Sinauer Associates, Sunderland, Massachusetts.

FINNERTY, J.R., PANG, K., BURTON, P., PAULSON, D. and MARTINDALE, M.Q. (2004). Origins of bilateral symmetry: HoX and Dpp expression in a sea anemone. Science 304: 1335-1337.

FREEMAN, G. (1981). The role of polarity in the development of the hydrozoan planula larva. Roux's Arch Dev Biol 190: 168-184.

FRITZENWANKER, J. H. and TECHNAU, U. (2002). Induction of gametogenesis in the basal cnidarian Nematostella vectensis (Anthozoa). Dev Genes Evol 212: 99-103.

GAVIS, E.R., CURTIS, D. and LEHMANN, R. (1996). Identification of cis-acting sequences that control nanos RNA localization. Dev Biol 176: 36-50.

GILBERT, S.F. (2003). Developmental Biology. Sinauer Associates, Sunderland, Massachusetts.

HAND, C. and UHLINGER, K.R. (1992). The culture, sexual and asexual reproduction and growth of the sea anemone Nematostella vectensis. Biol Bull 182: 169176.

KOBAYASHI, S., YAMADA, M., ASAOKA, M. and KITAMURA, T. (1996). Essential role of the posterior morphogen nanos for germline development in Drosophila. Nature 380: 708-711.

MARTINDALE, M.Q., PANG, K. and FINNERTY, J.R. (2004). Investigating the origins of triploblasty: "mesodermal" gene expression in a diploblastic animal, the sea anemone Nematostella vectensis (phylum, Cnidaria; class, Anthozoa). Development 131: 2463-2474.

MOCHIZUKI, K., ANO, H., KOBAYASI, S., NISHIMIYA-FUJISAWA, C. and FUJISAWA, T. (2000). Expression and evolutionary conservation of nanos related genes in Hydra. Dev Genes Evol 210: 591-602.

MOCHIZUKI, K., NISHIMIYA-FUJISAWA, C. and FUJISAWA, T. (2001). Universal occurrence of the vasa-related genes among metazoans and their germline expression in Hydra. Dev Genes Evol 211: 299-308.

NARDELLI-HAEFLIGER, D., BRUCE, A.E.E. and SHANKLAND, M. (1994). An axial domain of HOM/Hox gene expression is formed by morphogenetic alignment of independently specified cell lineages in the leech Helobdella. Development 120: 1839-1849.

SEIPEL, K., YANZE, N. and SCHMID, V. (2004). The germ line and somatic stem cell gene Cniwi in the jellyfish Podocoryne carnea. Int. J. Dev. Biol. 48: 1-7.

SCHOLZ, C. B. and TECHNAU, U. (2003). The ancestral role of Brachyury: expression of NemBra1 in the basal cnidarian Nematostella vectensis (Anthozoa). Dev Genes Evol 212: 563-570.

SCHWOERER-BÖHNING, B., KROIHER, M. and MÜLLER, W.A. (1990). Signal transmission and covert prepattern in the metamorphosis of Hydractinia echinata (Hydrozoa). Roux's Arch Dev Biol 198: 245-251.

TORRAS, R., YANZE, N., SCHMID, V. and GONZÁLEZ-CRESPO, S. (2004). nanos expression at the embryonic posterior pole and the medusa phase in the hydrozoan Podocoryne carnea. Evol \& Dev 6: 362-371.

YANZE, N., SPRING, J., SCHMIDLI, C. and SCHMID, V. (2001). Conservation of Hox/ParaHox-related genes in the early development of a cnidarian. Dev Biol 236: 89-98.

WANG, B.B., MUELLER-IMMERGLUCK, M.M., AUSTIN, J., ROBINSON, N.T., CHISHOLM, A. and KENYON, C. (1993). A homeotic gene cluster patterns the anteroposterior body axis of $C$. elegans. Cell 74: 29-42.

WANG, C. and LEHMAN, R. (1991). Nanos is the localized posterior determinant in Drosophila. Cell 66: 637-647. 